\title{
Theoretical and Experimental Values for the Rydberg Constant Do Not Match
}

\author{
Koshun Suto \\ Chudaiji Buddhist Temple, Isesaki, Japan \\ Email: koshun_suto129@mbr.nifty.com
}

How to cite this paper: Suto, K. (2021) Theoretical and Experimental Values for the Rydberg Constant Do Not Match. Journal of Applied Mathematics and Physics, 9, 1993-2003.

https://doi.org/10.4236/jamp.2021.98130

Received: June 26, 2021

Accepted: August 22, 2021

Published: August 25, 2021

Copyright (c) 2021 by author(s) and Scientific Research Publishing Inc. This work is licensed under the Creative Commons Attribution International License (CC BY 4.0).

http://creativecommons.org/licenses/by/4.0/ (c) (i) Open Access

\begin{abstract}
In many areas of physics and chemistry, the Rydberg constant is a fundamental physical constant that plays an important role. It comes into play as an indispensable physical constant in basic equations for describing natural phenomena. The Rydberg constant appears in the formula for calculating the wavelengths in the line spectrum emitted from the hydrogen atom. However, this Rydberg wavelength formula is a nonrelativistic formula derived at the level of classical quantum theory. In this paper, the Rydberg formula is rewritten as a wavelength formula taking into account the theory of relativity. When this is done, we come to an unexpected conclusion. What we try to determine by measuring spectra wavelengths is not actually the value of the Rydberg constant $R_{\infty}$ but the value $R_{n, m}$ of Formula (18). $R_{\infty}$ came into common use in the world of nonrelativistic classical quantum theory. If the theory of relativity is taken into account, $R_{\infty}$ can no longer be regarded as a physical constant. That is, we have continued to conduct experiments to this day in an attempt to determine the value of a physical constant, the Rydberg constant, which does not exist in the natural world.
\end{abstract}

\section{Keywords}

Rydberg Constant, Rydberg Formula, Classical Quantum Theory, Einstein's Energy-Momentum Relationship, Suto's Energy-Momentum Relationship

\section{Introduction}

In many areas of physics and chemistry, the Rydberg constant is a fundamental physical constant (abbreviated below as "physical constant") that plays an important role. It comes into play as an indispensable physical constant in basic equations for describing natural phenomena. Around the end of the $19^{\text {th }}$ century, Balmer, Rydberg, and others discovered that the following relation holds be- 
tween the wavelengths $\lambda$ of the emission line spectra of an atom.

$$
\frac{1}{\lambda}=R_{\infty}\left(\frac{1}{m^{2}}-\frac{1}{n^{2}}\right)
$$

This $R_{\infty}$ is the Rydberg constant. Its value is fixed, and does not depend on the spectra series or atom. The physical status of this empirical formula, which was obtained experimentally, was indicated by Bohr. In Bohr's theory of the hydrogen atom, $R_{\infty}$ is given by the following formula.

$$
R_{\infty}=\frac{2 \pi^{2} m_{\mathrm{e}} e^{4}}{c h^{3}}=10973731.568160(21) \mathrm{m}^{-1} \text {. (NIST CODATA } 2018 \text { value) }
$$

Here, it is shown that $R_{\infty}$ is an approximate value obtained by assuming that the mass of the proton is infinite. To obtain a value with higher precision, the mass of the electron must be replaced with the reduced mass of the electron and proton. This results in a correction of about $1 / 2000$. The correction due to the theory of Dirac, which treats the hydrogen atom relativistically, is on the order of $\alpha^{2}(\alpha \sim 1 / 137)$. Furthermore, the correction of quantum electrodynamics (QED) is on the order of $\alpha^{3}$.

A crucial point is that all of these corrections vary in proportion to $R_{\infty}$. Therefore, even when these corrections are made, it is thought that the value of $R_{\infty}$ can be found with high precision.

In the classical quantum theory of Bohr, the energy levels of the hydrogen atom are given by the following formula [1].

$$
\begin{aligned}
E_{\mathrm{BO}, n} & =-\frac{1}{2}\left(\frac{1}{4 \pi \varepsilon_{0}}\right)^{2} \frac{m_{\mathrm{e}} e^{4}}{\hbar^{2}} \cdot \frac{1}{n^{2}} \\
& =-\frac{\alpha^{2} m_{\mathrm{e}} c^{2}}{2 n^{2}}, \quad n=1,2, \cdots .
\end{aligned}
$$

Here, $E_{\text {Во }}$ refers to the total mechanical energy predicted by Bohr. Also, $\alpha$ is the following fine-structure constant.

$$
\alpha=\frac{e^{2}}{4 \pi \varepsilon_{0} \hbar c}=7.2973525693 \times 10^{-3} .
$$

The photonic energy emitted during a transition between energy levels $\left(E_{\mathrm{BO}, n}-E_{\mathrm{BO}, m}\right)$ and wavelength $\lambda_{n, m}$ for principal quantum numbers $n$ and $m$ can be expressed as follows.

$$
\begin{aligned}
& E_{\mathrm{BO}, n}-E_{\mathrm{BO}, m}=h v=\frac{h c}{\lambda_{n, m}} \\
& =h c R_{\infty}\left(\frac{1}{m^{2}}-\frac{1}{n^{2}}\right), \quad m=1,2, \cdots ; n=m+1, m+2, \cdots .
\end{aligned}
$$

The Rydberg formula can be derived from Formula (5) as indicated below.

$$
\frac{1}{\lambda_{n, m}}=\frac{E_{\mathrm{BO}, n}-E_{\mathrm{BO}, m}}{h c}=R_{\infty}\left(\frac{1}{m^{2}}-\frac{1}{n^{2}}\right), \quad m=1,2, \cdots ; n=m+1, m+2, \cdots .
$$

Formula (6) is derived from Formula (3), and thus is not a wavelength formu- 
la taking into account the theory of relativity.

Incidentally, the author has already derived the following energy levels of the hydrogen atom taking into account the theory of relativity (Appendix A).

$$
\begin{aligned}
E_{\mathrm{re}, n} & =m_{n} c^{2}-m_{\mathrm{e}} c^{2}=m_{\mathrm{e}} c^{2}\left[\left(1+\frac{\alpha^{2}}{n^{2}}\right)^{-1 / 2}-1\right] \\
& =m_{\mathrm{e}} c^{2}\left[\frac{n}{\left(n^{2}+\alpha^{2}\right)^{1 / 2}}-1\right], n=1,2, \cdots .
\end{aligned}
$$

Here, $m_{n} c^{2}$ is the relativistic energy of the electron when the principal quantum number is in the state $n$.

This paper defines $E_{\mathrm{re}, n}$ as the relativistic energy levels of the hydrogen atom derived at the level of classical quantum theory. (The quantum number used here is just the principal quantum number. Therefore, $E_{\mathrm{re}, n}$ is not a formula which predicts all the relativistic energy levels of the hydrogen atom.)

However, the term "relativistic" used here does not mean based on the special theory of relativity (STR). It means that the expression takes into account the fact that the mass of the electron varies due to velocity. According to the STR, the electron's mass increases when its velocity increases. However, inside the hydrogen atom, the mass of the electron decreases when the velocity of the electron increases. Attention must be paid to the fact that, inside the hydrogen atom, the relativistic mass of the electron $m_{n}$ is smaller than the rest mass $m_{\mathrm{e}}$.

The following figures the energy levels of the hydrogen atom derived by Bohr, and the energy levels derived by the author (Figure 1).

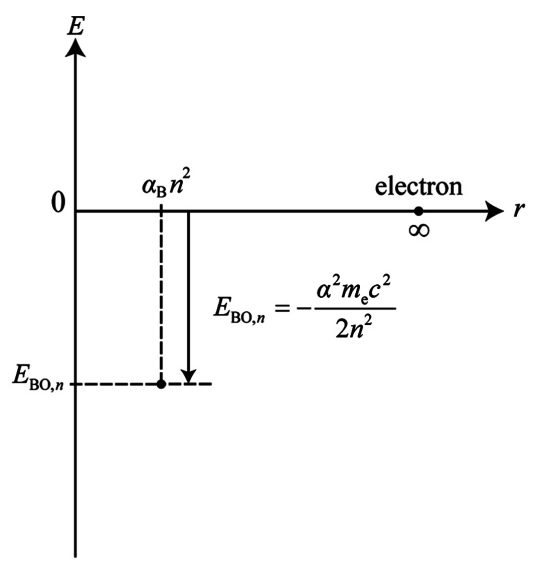

(a)

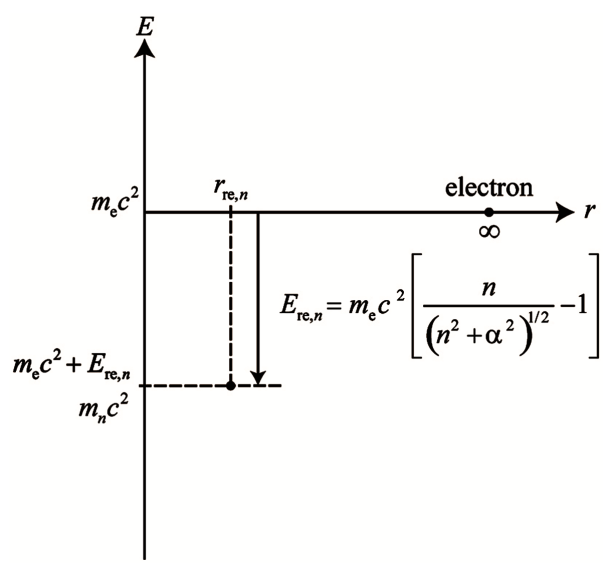

(b)

Figure 1. (a) In Bohr's theory, the energy when the electron is at rest at a position infinitely distant from the proton (atomic nucleus) is defined to be zero. Formula (3) does not give energy levels derived from an absolute scale. (b) According to the STR, the energy of an electron at rest at a position where $r=\infty$ is $m_{\mathrm{e}} c^{2} . E_{\mathrm{re}, n}$ is given by the difference between $m_{\mathrm{e}} c^{2}$ and $m_{n} c^{2}$ the relativistic energy of the electron described with an absolute scale. Therefore, $E_{\mathrm{re}, n}$ are also energy levels described from an absolute perspective. 
Now, if a Taylor expansion is performed on the right side of Formula (7a),

$$
\begin{aligned}
E_{\mathrm{re}, n} & =m_{\mathrm{e}} c^{2}\left[\left(1-\frac{\alpha^{2}}{2 n^{2}}+\frac{3 \alpha^{4}}{8 n^{4}}-\frac{5 \alpha^{6}}{16 n^{6}}+\cdots\right)-1\right] \\
& =-\frac{\alpha^{2} m_{\mathrm{e}} c^{2}}{2 n^{2}}\left(1-\frac{3 \alpha^{2}}{4 n^{2}}+\frac{5 \alpha^{4}}{8 n^{4}}-\cdots\right) \\
& =E_{\mathrm{BO}, n}\left(1-\frac{3 \alpha^{2}}{4 n^{2}}+\frac{5 \alpha^{4}}{8 n^{4}}-\cdots\right) .
\end{aligned}
$$

Comparing Formulas ( $8 \mathrm{~b}$ ) and (3b), it is evident that Formula (3) is an approximation of Formula (7). That is,

$$
E_{\mathrm{re}, n} \approx E_{\mathrm{BO}, n} \text {. }
$$

Now, the author has already shown that the following wavelength formula can be derived from Formula (7) [2] (Appendix B)

$$
\begin{aligned}
& \frac{1}{\lambda_{n, m}}=\frac{E_{\mathrm{re}, n}-E_{\mathrm{re}, m}}{h c} \\
& =\frac{1}{\lambda_{\mathrm{C}}}\left[\left(1+\frac{\alpha^{2}}{n^{2}}\right)^{-1 / 2}-\left(1+\frac{\alpha^{2}}{m^{2}}\right)^{-1 / 2}\right], m=1,2, \cdots ; n=m+1, m+2, \cdots .
\end{aligned}
$$

Formula (10) is a wavelength formula taking the theory of relativity into account. In this paper, Formula (10) is rewritten in a form similar to Formula (6). It is also checked what happens to the part corresponding to $R_{\infty}$ in Formula (6) in the newly derived formula.

\section{The Relativistic Wavelength Formula Obtained by Rewriting Formula (10)}

If the Taylor expansion of Formula (10) is taken, the following formula is obtained.

$$
\begin{aligned}
& \frac{1}{\lambda_{n, m}}=\frac{1}{\lambda_{\mathrm{C}}}\left[\left(1-\frac{\alpha^{2}}{2 n^{2}}+\frac{3 \alpha^{4}}{8 n^{4}}-\frac{5 \alpha^{6}}{16 n^{6}}+\cdots\right)-\left(1-\frac{\alpha^{2}}{2 m^{2}}+\frac{3 \alpha^{4}}{8 m^{4}}-\frac{5 \alpha^{6}}{16 m^{6}}+\cdots\right)\right] \\
= & \frac{\alpha^{2}}{2 \lambda_{\mathrm{C}}} \cdot \frac{2}{\alpha^{2}}\left[\left(1-\frac{\alpha^{2}}{2 n^{2}}+\frac{3 \alpha^{4}}{8 n^{4}}-\frac{5 \alpha^{6}}{16 n^{6}}+\cdots\right)-\left(1-\frac{\alpha^{2}}{2 m^{2}}+\frac{3 \alpha^{4}}{8 m^{4}}-\frac{5 \alpha^{6}}{16 m^{6}}+\cdots\right)\right] .
\end{aligned}
$$

The following relationship is used here.

$$
R_{\infty}=\frac{2 \pi^{2} m_{\mathrm{e}} e^{4}}{c h^{3}}=\frac{\alpha^{2} m_{\mathrm{e}} c}{2 h}=\frac{\alpha^{2}}{2 \lambda_{\mathrm{C}}} .
$$

Formula (11b) can then be written as follows.

$$
\begin{gathered}
\frac{1}{\lambda_{n, m}}=R_{\infty} \\
\cdot \frac{2}{\alpha^{2}}\left[\left(1-\frac{\alpha^{2}}{2 n^{2}}+\frac{3 \alpha^{4}}{8 n^{4}}-\frac{5 \alpha^{6}}{16 n^{6}}+\cdots\right)-\left(1-\frac{\alpha^{2}}{2 m^{2}}+\frac{3 \alpha^{4}}{8 m^{4}}-\frac{5 \alpha^{6}}{16 m^{6}}+\cdots\right)\right] \\
=R_{\infty}\left[\left(\frac{1}{m^{2}}-\frac{3 \alpha^{2}}{4 m^{4}}+\frac{5 \alpha^{4}}{8 m^{6}}-\cdots\right)-\left(\frac{1}{n^{2}}-\frac{3 \alpha^{2}}{4 n^{4}}+\frac{5 \alpha^{4}}{8 n^{6}}-\cdots\right)\right]
\end{gathered}
$$




$$
\approx R_{\infty}\left(\frac{1}{m^{2}}-\frac{1}{n^{2}}\right)+R_{\infty}\left(\frac{3 \alpha^{2}}{4 n^{4}}-\frac{3 \alpha^{2}}{4 m^{4}}-\frac{5 \alpha^{4}}{8 n^{6}}+\frac{5 \alpha^{4}}{8 m^{6}}\right) .
$$

It is evident from this that the previously-known Formula (6) is an approximation of Formula (10).

Next, Formulas (6) and (10) are further compared. If, in Formula (10), the values of the Compton wavelength $\lambda_{\mathrm{C}}$ and fine structure constant $\alpha$ are determined, it is possible to calculate the spectra wavelengths. Of course, Formula (6) too must predict the spectra wavelengths. However, at present, the spectra wavelengths are first measured, and then the value of $R_{\infty}$ is determined based on those values. Formula (6) is not for calculating wavelengths, but for determining the Rydberg constant.

Next, the following table summarizes the wavelengths calculated using the Rydberg Formula (6) and Formula (10) for wavelengths derived by the author (Table 1).

The discussion thus far has already been presented in another paper (reference [2]). The discussion presented for the first time in this paper begins here.

Formula (10) is first rewritten as the following formula similar to Formula (6).

$$
\frac{1}{\lambda_{n, m}}=R\left(\frac{1}{m^{2}}-\frac{1}{n^{2}}\right), \quad R \neq R_{\infty} .
$$

First, Formula (10) can be written as follows taking Formula (12) into account.

$$
\frac{1}{\lambda_{n, m}}=R_{\infty} \cdot \frac{2}{\alpha^{2}}\left[\frac{n}{\left(n^{2}+\alpha^{2}\right)^{1 / 2}}-\frac{m}{\left(m^{2}+\alpha^{2}\right)^{1 / 2}}\right] .
$$

Also, Formula (15) can be written as follows.

$$
\frac{1}{\lambda_{n, m}}=R_{\infty} \cdot \frac{2}{\alpha^{2}}\left[\frac{n}{\left(n^{2}+\alpha^{2}\right)^{1 / 2}}-\frac{m}{\left(m^{2}+\alpha^{2}\right)^{1 / 2}}\right] \frac{m^{2} n^{2}}{n^{2}-m^{2}}\left(\frac{1}{m^{2}}-\frac{1}{n^{2}}\right) .
$$

However,

$$
\frac{m^{2} n^{2}}{n^{2}-m^{2}}\left(\frac{1}{m^{2}}-\frac{1}{n^{2}}\right)=1
$$

Here, the following $R_{n, m}$ is defined.

Table 1. Wavelength values predicted by Bohr's theory and this paper. The following values of CODATA were used when calculating energies. $\lambda_{\mathrm{C}}=2.42631023867 \times 10^{-12} \mathrm{~m}$.

\begin{tabular}{cccc}
\hline & Rydberg Formula (Formula (6)) & This paper (Formula (10)) & Remark \\
\hline$\lambda_{2,1}$ & $121.502 \mathrm{~nm}$ & $121.506 \mathrm{~nm}$ & Lyman $\alpha$ \\
$\lambda_{3,1}$ & $1.02518 \mathrm{~nm}$ & $1.02547 \mathrm{~nm}$ & Lyman $\beta$ \\
$\lambda_{3,2}$ & $656.112 \mathrm{~nm}$ & $656.123 \mathrm{~nm}$ & \\
$\lambda_{4,2}$ & $486.009 \mathrm{~nm}$ & $486.015 \mathrm{~nm}$ & \\
\hline
\end{tabular}




$$
\begin{aligned}
R_{n, m} & =R_{\infty} \cdot \frac{2}{\alpha^{2}} \cdot \frac{m^{2} n^{2}}{n^{2}-m^{2}}\left[\frac{n}{\left(n^{2}+\alpha^{2}\right)^{1 / 2}}-\frac{m}{\left(m^{2}+\alpha^{2}\right)^{1 / 2}}\right] \\
& =\frac{1}{\lambda_{\mathrm{C}}} \cdot \frac{m^{2} n^{2}}{n^{2}-m^{2}}\left[\frac{n}{\left(n^{2}+\alpha^{2}\right)^{1 / 2}}-\frac{m}{\left(m^{2}+\alpha^{2}\right)^{1 / 2}}\right] .
\end{aligned}
$$

As is clear from Formula (18), $R_{n, m}$ is not a constant. Thus, in Formula (18), $R$ has subscripts $n, m$.

If $R_{n, m}$ is defined similar to Formula (18), then Formula (14) can be written as follows.

$$
\begin{aligned}
\frac{1}{\lambda_{n, m}} & =\frac{1}{\lambda_{\mathrm{C}}} \cdot \frac{m^{2} n^{2}}{n^{2}-m^{2}}\left[\frac{n}{\left(n^{2}+\alpha^{2}\right)^{1 / 2}}-\frac{m}{\left(m^{2}+\alpha^{2}\right)^{1 / 2}}\right]\left(\frac{1}{m^{2}}-\frac{1}{n^{2}}\right) \\
& =R_{n, m}\left(\frac{1}{m^{2}}-\frac{1}{n^{2}}\right), \quad m=1,2, \cdots ; n=m+1, m+2, \cdots
\end{aligned}
$$

Here, if $R_{\infty}$ and $R_{n, m}$ are compared,

$$
\frac{R_{n, m}}{R_{\infty}}=\frac{2}{\alpha^{2}} \cdot \frac{m^{2} n^{2}}{n^{2}-m^{2}}\left[\frac{n}{\left(n^{2}+\alpha^{2}\right)^{1 / 2}}-\frac{m}{\left(m^{2}+\alpha^{2}\right)^{1 / 2}}\right] .
$$

It is thus evident that the value we try to determine by measuring spectra wavelengths is not $R_{\infty}$ in Formula (6) but $R_{n, m}$ in Formula (19b). However, that fact has not been noticed by anyone thus far. Furthermore, $R_{n, m}$ is not a physical constant, as is evident from Formula (18).

Next, Table 2 summarizes the values of $R_{n, m}$ when $m=1,2$ and $n=2,3$.

Ordinarily, we determine the value of the Rydberg constant in Formula (6) by measuring the spectra wavelengths. If this value matches with the theoretical value (Formula (2)), then the validity of Bohr's model of the atom is confirmed.

However, the value we try to determine through experiment is the value of $R_{n, m}$ not the value of the Rydberg constant $R_{\infty}$. Therefore, if more precise measurement becomes possible, it will become clear that the theoretical value (Formula (2)) and experimental value (Formula (18)) do not match exactly.

Originally, Formula (1) is given as follows.

Table 2. Relation between the Rydberg constant $R_{\infty}$ and $R_{n, m}$. The values of $R_{\infty}$ and $R_{n, m}$ are almost equal, but it is always the case that $R_{n, m}<R_{\infty}$.

\begin{tabular}{ccc}
\hline & $R_{n, m} / R_{\infty}$ & Theoretical value of $R_{n, m}$ \\
\hline$R_{2,1}$ & 0.999969406 & $10973395.8 \mathrm{~m}^{-1}$ \\
$R_{3,1}$ & 0.999712063 & $10970571.8 \mathrm{~m}^{-1}$ \\
$R_{3,2}$ & 0.999984387 & $10973560.3 \mathrm{~m}^{-1}$ \\
\hline
\end{tabular}




$$
\frac{1}{\lambda_{n . m}}=\frac{2 \pi^{2} m_{\mathrm{e}} e^{4}}{c h^{3}}\left(\frac{1}{m^{2}}-\frac{1}{n^{2}}\right) .
$$

However, writing out the right side of Formula (2) is bothersome, and for reasons of convenience, this expression was replaced with the single symbol $R_{\infty}$. (However, this is not a description of the actual history.) Therefore, $R_{\infty}$ is not a physical constant on a par with $c$ or $e$. It is also not the case that we discovered a physical constant $R_{\infty}$. $R_{\infty}$ came into common use in the world of nonrelativistic classical quantum theory. If the theory of relativity is taken into account, $R_{\infty}$ can no longer be regarded as a physical constant.

\section{Conclusions}

The formula for wavelengths in classical quantum theory is the following.

$$
\frac{1}{\lambda_{n, m}}=R_{\infty}\left(\frac{1}{m^{2}}-\frac{1}{n^{2}}\right), m=1,2, \cdots ; n=m+1, m+2, \cdots .
$$

In contrast, the author has previously derived the following formula, more precise than Formula (22), by taking into account the theory of relativity.

$$
\frac{1}{\lambda_{n, m}}=\frac{1}{\lambda_{\mathrm{C}}}\left[\frac{n}{\left(n^{2}+\alpha^{2}\right)^{1 / 2}}-\frac{m}{\left(m^{2}+\alpha^{2}\right)^{1 / 2}}\right], \quad m=1,2, \cdots ; n=m+1, m+2, \cdots .
$$

Formula (22) is an approximation of Formula (23). Formula (23) can be rewritten as the following formula similar to Formula (22).

$$
\frac{1}{\lambda_{n, m}}=R_{n, m}\left(\frac{1}{m^{2}}-\frac{1}{n^{2}}\right), \quad m=1,2, \cdots ; n=m+1, m+2, \cdots .
$$

At this time, $R_{n, m}$ is defined as follows.

$$
R_{n, m}=\frac{1}{\lambda_{\mathrm{C}}} \cdot \frac{m^{2} n^{2}}{n^{2}-m^{2}}\left[\frac{n}{\left(n^{2}+\alpha^{2}\right)^{1 / 2}}-\frac{m}{\left(m^{2}+\alpha^{2}\right)^{1 / 2}}\right], m=1,2, \cdots ; n=m+1, m+2, \cdots .
$$

What we try to determine by measuring spectra wavelengths is not actually the value of the Rydberg constant $R_{\infty}$ but the value $R_{n, m}$ of Formula (25). However, $R_{n, m}$ is not a physical constant. That is, we have continued to conduct experiments to this day in an attempt to determine the value of a physical constant, the Rydberg constant, which does not exist in the natural world.

\section{Acknowledgements}

I would like to express my thanks to the staff at ACN Translation Services for their translation assistance. Also, I wish to express my gratitude to Mr. H. Shimada for drawing figures.

\section{Conflicts of Interest}

The author declares no conflicts of interest regarding the publication of this paper. 


\section{References}

[1] Bohr, N. (1913) On the Constitution of Atoms and Molecules. Philosophical Magazine, 26, 1. https://doi.org/10.1080/14786441308634955

[2] Suto, K. (2020) Relativistic Correction of the Rydberg Formula. Journal of Modern Physics, 11, 294-303. https://doi.org/10.4236/jmp.2020.112018

[3] Sommerfeld, A. (1923) Atomic Structure and Spectral Lines. Methuen \& Co. Ltd., London, 528.

[4] Suto, K. (2019) The Relationship Enfolded in Bohr's Quantum Condition and a Previously Unknown Formula for Kinetic Energy. Applied Physics Research, 11, 19-34. https://doi.org/10.5539/apr.v11n1p19

[5] Suto, K. (2011) An Energy-Momentum Relationship for a Bound Electron inside a Hydrogen Atom. Physics Essays, 24, 301-307. https://doi.org/10.4006/1.3583810

[6] Suto, K. (2020) Theoretical Prediction of Negative Energy Specific to the Electron. Journal of Modern Physics, 11, 712-724. https://doi.org/10.4236/jmp.2020.115046

[7] Suto, K. (2021) The Quantum Condition That Should Have Been Assumed by Bohr When Deriving the Energy Levels of a Hydrogen Atom. Journal of Applied Mathematics and Physics, 9, 1230-1244. https://doi.org/10.4236/jamp.2021.96084 


\section{Appendix A}

The energy-momentum relationship in the STR holds in an isolated system in free space. Here, if $m_{0} c^{2}$ is the rest mass energy and $m c^{2}$ is the relativistic energy, the relationship can be written as follows.

$$
\left(m_{0} c^{2}\right)^{2}+p^{2} c^{2}=\left(m c^{2}\right)^{2}
$$

The following equation holds due to Formula (A1).

$$
\left(m c^{2}\right)^{2}=m_{0}^{2} c^{4}+\left(m^{2} c^{4}-m_{0}^{2} c^{4}\right)=\left(m_{0} c^{2}\right)^{2}+\left(m+m_{0}\right)\left(m c^{2}-m_{0} c^{2}\right) c^{2} .
$$

Using this, Formula (A1) becomes as follows.

$$
\left(m_{0} c^{2}\right)^{2}+\left(m+m_{0}\right)\left(m c^{2}-m_{0} c^{2}\right) c^{2}=\left(m c^{2}\right)^{2} .
$$

Incidentally, Sommerfeld and Einstein defined relativistic kinetic energy as follows [3].

$$
K_{\mathrm{re}}=m c^{2}-m_{0} c^{2} .
$$

Since Formulas (A1) and Formula (A3) are equal, the following relationship must hold when Formula (A4) is taken into account.

$$
p^{2}=\left(m_{0}+m\right)\left(m c^{2}-m_{0} c^{2}\right)=\left(m_{0}+m\right) K_{\mathrm{re}} .
$$

The following formula is obtained from this.

$$
K_{\mathrm{re}}=\frac{p^{2}}{m+m_{0}},
$$

Formula (A6) is the formula for relativistic kinetic energy. Classical (non-relativistic) kinetic energy, in contrast, is defined as follows.

$$
K_{\mathrm{cl}}=\frac{1}{2} m_{0} v^{2}=\frac{p^{2}}{2 m_{0}} .
$$

Formula (A6) describes the relativistic kinetic energy of an electron in a hydrogen atom, and Formula (A7) describes the classical kinetic energy of an electron.

Next, the relativistic kinetic energy of an electron in a hydrogen atom is defined as follows by referring to Formula (A6) [4].

$$
K_{\mathrm{re}, n}=\frac{p_{\mathrm{re}, n}^{2}}{m_{\mathrm{e}}+m_{n}}, \quad p_{\mathrm{re}, n}=m_{n} v_{n} \text {. }
$$

Here, $m_{n}$ is the relativistic mass of the electron. Also, $p_{\mathrm{re}, n}$ indicates the relativistic momentum of the electron.

Incidentally, the energy of an electron at rest in an isolated system in free space is $m_{\mathrm{e}} c^{2}$. Here, we consider the case where this electron is drawn in by the electrical attraction of the proton, and forms a hydrogen atom. At this time, the electron emits a photon to the outside. Therefore, the relativistic energy of an electron in a hydrogen atom $m_{n} c^{2}$ becomes smaller than the rest mass energy $m_{\mathrm{e}} c^{2}$. That is, 


$$
m_{n} c^{2}<m_{\mathrm{e}} c^{2} .
$$

The behavior of an electron inside an atom, where there is potential energy, cannot be described with the relationship of Einstein (A1). Caution is necessary because it is completely overlooked in Formula (A9).

Now, referring to Formula (A4), it is natural to define the relativistic kinetic energy of an electron in a hydrogen atom as follows.

$$
K_{\mathrm{re}, n}=-E_{\mathrm{re}, n}=m_{\mathrm{e}} c^{2}-m_{n} c^{2} .
$$

In this way, two formulas have been obtained for the relativistic kinetic energy of the electron in a hydrogen atom (Formulas (A8), and (A10)).

Incidentally, the following equation can be derived from Formulas (A8) and (A10).

$$
\frac{p_{\mathrm{re}, n}^{2}}{m_{\mathrm{e}}+m_{n}}=m_{\mathrm{e}} c^{2}-m_{n} c^{2} .
$$

Rearranging this, the following relationship can be derived.

$$
\left(m_{n} c^{2}\right)^{2}+p_{\mathrm{re}, n}^{2} c^{2}=\left(m_{\mathrm{e}} c^{2}\right)^{2} .
$$

Formula (A12) is the energy-momentum relationship applicable to the electron in a hydrogen atom. (The author calls this "Suto's energy-momentum relationship".)

The author has already derived Formula (A12) using three methods [5] [6] [7]. Here, the method derived in Reference [7] is presented.

The relation between $m_{n}$ and $m_{\mathrm{e}}$ is as follows due to Formula (A12).

$$
m_{n}=m_{\mathrm{e}}\left(1+\frac{\alpha^{2}}{n^{2}}\right)^{-1 / 2} \text {. }
$$

When deriving Formula (A13) from Formula (A12), the following already-known relation was used [7].

$$
\frac{v_{n}}{c}=\frac{\alpha}{n} .
$$

Hence, the energy levels of a hydrogen atom $E_{\mathrm{re}, n}$ are:

$$
\begin{aligned}
E_{\mathrm{re}, n} & =-K_{\mathrm{re}, n}=m_{n} c^{2}-m_{\mathrm{e}} c^{2}=m_{\mathrm{e}} c^{2}\left[\left(1+\frac{\alpha^{2}}{n^{2}}\right)^{-1 / 2}-1\right] \\
& =m_{\mathrm{e}} c^{2}\left[\frac{n}{\left(n^{2}+\alpha^{2}\right)^{1 / 2}}-1\right], \quad n=1,2, \cdots .
\end{aligned}
$$

\section{Appendix B}

The differences in energy between different energy levels in the hydrogen atom can be found with the following formula [2].

$$
E_{\mathrm{re}, n}-E_{\mathrm{re}, m}=\left(m_{n} c^{2}-m_{\mathrm{e}} c^{2}\right)-\left(m_{m} c^{2}-m_{\mathrm{e}} c^{2}\right)=m_{n} c^{2}-m_{m} c^{2}
$$




$$
=m_{\mathrm{e}} c^{2}\left[\left(1+\frac{\alpha^{2}}{n^{2}}\right)^{-1 / 2}-\left(1+\frac{\alpha^{2}}{m^{2}}\right)^{-1 / 2}\right], \quad n=m+1, m+2, \cdots
$$

The following equation is also known.

$$
\lambda_{\mathrm{C}}=\frac{h}{m_{\mathrm{e}} c} .
$$

Here, $\lambda_{\mathrm{C}}$ is the Compton wavelength of the electron.

Taking into account Formula (B2),

$$
m_{\mathrm{e}} c^{2}=\frac{h c}{\lambda_{\mathrm{C}}} .
$$

Based on this, Formula (B1) can be written as follows [2].

$$
\begin{aligned}
& \frac{1}{\lambda_{n, m}}=\frac{E_{\mathrm{re}, n}-E_{\mathrm{re}, m}}{h c} \\
& =\frac{1}{\lambda_{\mathrm{C}}}\left[\left(1+\frac{\alpha^{2}}{n^{2}}\right)^{-1 / 2}-\left(1+\frac{\alpha^{2}}{m^{2}}\right)^{-1 / 2}\right], \quad m=1,2, \cdots ; n=m+1, m+2, \cdots .
\end{aligned}
$$

Formula (B4) is the formula for wavelength, taking into account the theory of relativity. 